\title{
La responsabilidad social de las cooperativas en Cuba. Limitaciones y oportunidades
}

\author{
Yamira Mirabal González' \\ Universidad de Pinar del Río, Cuba
}

Sumario: I. Introducción. II. La Responsabilidad Social Empresarial. III. La Responsabilidad Social Cooperativa. IV. Procedimiento para la realización de la Responsabilidad Social Cooperativa. V. Limitaciones y resultados de la Responsabilidad Social Cooperativa en Cuba. VI. Conclusiones.

Resumen: El sector cooperativo manifiesta un conjunto de limitaciones en su proceso de gestión, que desvirtúan su naturaleza y esencia cooperativa y limitan la práctica de su Responsabilidad Social.

Atendiendo a que las empresas sostenibles se gestionan en función de las dimensiones económicas, sociales y ambientales, este trabajo persigue como objetivo, fundamentar la práctica de la Responsabilidad Social Cooperativa en Cuba, a partir de la implementación de un procedimiento que se corresponde con tales fines. Para ello se abordan los fundamentos teóricos de la Responsabilidad Social Empresarial y la Responsabilidad Social Cooperativa. Por último se presentan las limitaciones que obstaculizan la práctica de la Responsabilidad Social Cooperativa y los resultados identificados en función del desarrollo y consolidación de dicho proceso en el país.

Palabras clave: cooperativa, responsabilidad social cooperativa.

Abstract: The cooperative sector manifests a set of limitations in its management process, which distort its cooperative nature and essence and limit the practice of its Social Responsibility.

Taking into account that sustainable companies are managed according to economic, social and environmental dimensions, this work aims to support the practice of Cooperative Social Responsibility in Cuba, based on the implementation of a procedure that corresponds to these purposes. For this, the theoretical foundations of Corporate Social Responsibility and Cooperative Social Responsibility are addressed. Finally, the limitations that hinder

1 Doctora en Ciencias Contables y Financieras. Profesora Auxiliar del Centro de Estudios de Dirección, Desarrollo Local, Turismo y Cooperativismo de la Universidad de Pinar del Río, Cuba. 
the practice of Cooperative Social Responsibility and the results identified according to the development and consolidation of said process in the country are presented.

Keywords: cooperative, cooperative social responsibility. 


\section{Introducción}

El desarrollo y consolidación del Sector de la Economía Social y Solidaria, y dentro de este las cooperativas como parte determinante del mismo en América Latina, así como la defensa política de estas organizaciones está condicionado a la observación más estricta de su dimensión social sobre la base de procesos económicos eficientes y eficaces. Las cooperativas deben demostrar con su desempeño diario las razones de su existencia como forma de gestión con peculiaridades que las distinguen del resto de las empresas, en función del manejo adecuado de sus dimensiones económicas y sociales.

En este sentido la COMISIÓN DE LAS COMUNIDADES EUROPEAS $(C C E)^{2}$ indicó que: "Las cooperativas tienen una larga tradición en combinar viabilidad económica y responsabilidad social gracias al diálogo entre las partes interesadas y a la gestión participativa, y pueden servir de referencia a otras organizaciones».

En el caso de Cuba, que se encuentra en un momento crucial para su desarrollo, donde se lleva a cabo un proceso de actualización de su Modelo Económico y Social, el sector cooperativo constituye uno de los principales protagonistas, sustentado en los aportes que en el orden económico y social, el mismo ha realizado en función de la elevación de los niveles de eficiencia y eficacia económico-productiva y del nivel y calidad de vida de sus miembros y de la sociedad, lo que reafirma su papel como actor clave del desarrollo local.

En Cuba el cooperativismo se desarrolla actualmente en una parte considerable de la agricultura, esfera decisiva para el desarrollo socioeconómico del país existiendo actualmente 4828 cooperativas distribuidas en las diferentes formas de organización de la producción agropecuaria (Cooperativas de Créditos y Servicios (CCS), Cooperativas de Producción Agropecuaria (CPA) y Unidades Básicas de Producción Cooperativa (UBPC), y en los servicios donde coexisten 398 Cooperativas no Agropecuarias (CNA) concentrándose el 89\% de estas, en cinco sectores: gastronomía, comercio, construcción, industria y servicios técnicos personales y del hogar.

A pesar de los resultados alcanzados por el cooperativismo cubano, aún se manifiestan insuficiencias en su proceso de gestión, dentro de las que se destacan las que afectan la esencia y naturaleza cooperativa, y que se relacionan con la no conjugación de sus objetivos económicos

2 COMISIÓN DE LAS COMUNIDADES EUROPEAS (CCE). Comunicación de la Comisión relativa a la responsabilidad social de las empresas: una contribución empresarial al desarrollo sostenible. Bruselas: CCE, 2002, pp. 11. 
y sociales donde se han priorizado los primeros como parte del proceso de gestión, y la limitada práctica de la Responsabilidad Social.

En este sentido, se realiza el presente trabajo que persigue como objetivo, fundamentar la práctica de la Responsabilidad Social Cooperativa en Cuba, a partir de la implementación de un procedimiento. Para ello se abordan los fundamentos teóricos de la Responsabilidad Social Empresarial y la Responsabilidad Social Cooperativa. Por último se presentan las limitaciones que obstaculizan la práctica de la Responsabilidad Social Cooperativa y los resultados identificados en función del desarrollo y consolidación de dicho proceso en el país.

\section{La Responsabilidad Social Empresarial}

Históricamente se concibió la responsabilidad de las empresas únicamente en función de la generación de utilidades, concepción que deja de ser aceptable, a partir de su evolución hacia el análisis de la repercusión favorable y desfavorable que tienen sus actividades económico-productivas en la calidad de vida de sus empleados y de las comunidades en las que estas se insertan.

Como consecuencia, las empresas comienzan a reconocer la responsabilidad social como un tema crucial para el desarrollo empresarial desde una perspectiva sistémica, no solo a partir de sus compromisos con los empleados, sino también desde sus vínculos con los sectores con los cuales se relacionan (comunidad, clientes, consumidores, proveedores, sociedad y estado) en los ámbitos de actuación económico, social y medioambiental entre otros y buscando la protección del medio ambiente y la sustentabilidad de las futuras generaciones.

(...) A la par de este proceso, a nivel internacional ha recobrado interés el estudio de términos tales como: 1) Responsabilidad Social Corporativa (RSC), 2) Ciudadanía Corporativa, 3) Sostenibilidad Empresarial (SE) y 4) Responsabilidad Social Empresarial (RSE), predominando el uso de los tres primeros en los países desarrollados y el cuarto en el contexto latinoamericano $(\ldots)^{3}$, presentándose diferentes acepciones sobre estos conceptos que para estudiosos del tema pueden ser considerados afines. A los efectos de esta investigación se utiliza el término Responsabilidad Social Empresarial.

La Responsabilidad Social Empresarial es un conjunto de prácticas de la organización que forman parte de su estrategia corporativa,

3 BETANCOURT ABIO, R. La responsabilidad social empresarial en Cuba. Revista Estudios del Desarrollo Social: Cuba y América Latina, vol. 4, no. 2, 2016, pp. 3. 
y que tienen como fin evitar daños y/o producir beneficios para todas las partes interesadas en la actividad de la empresa (clientes, empleados, accionistas, comunidad, entorno, etc.), siguiendo fines racionales y que deben redondear en un beneficio tanto para la organización como para la sociedad 4 .

El concepto de responsabilidad social empresarial debe entenderse como parte de un proceso de transformación evolutivo que el mundo empresarial ha tenido que llevar a cabo para pasar de sociedad industrial antigua, a una más actual, globalizada y responsable, consciente y comprometida con la conservación del medio ambiente y de la sociedad en su conjunto 5 .

La RSE es el compromiso consciente y congruente de cumplir integralmente con la finalidad de la empresa, tanto en lo interno como en lo externo, considerando las expectativas económicas, sociales y ambientales de todos sus participantes, demostrando respeto por la gente, los valores éticos, la comunidad y el medio ambiente, contribuyendo así a la construcción del bien común ${ }^{6}$.

La responsabilidad social empresarial es el compromiso continuo de contribuir al desarrollo económico sostenible, mejorando la calidad de vida de los empleados y sus familias, así como la de la comunidad local y de la sociedad en general ${ }^{7}$.

La RSE no solo está enfocada en la generación de valor agregado o a la obtención de mejores resultados financieros, sino que adicionalmente compromete en su atmósfera a actores internos como los colaboradores y accionistas, y actores externos como clientes, proveedores y distribuidores, considera que el rendimiento económico permite a una empresa crecer, la gestión de sus procesos y su transparencia le permiten sostenibilidad, es decir, existencia en el tiempo ${ }^{8}$.

4 VALLAEYS, F. ¿Qué es la responsabilidad social universitaria. Nuevo León, México. consultado en: www.cedus.cl, 2008, pp 1-2.

5 VÉLEZ EVANS, A.M. Una respuesta a la acción social, de la rentabilidad a la responsabilidad social empresarial. Revista Ciencias Estratégicas, vol. 18, no. 23, 2010, pp. 12.

6 CAJIGA CALDERÓN, J.F. El concepto de Responsabilidad Social Empresarial RSE. Centro Mexicano para la filantropía, 2015, pp. 4.

7 ANTELO-GONZÁLEZ, Y. y ALFONSO-ROBAINA, D. Análisis de la Responsabilidad Social Empresarial basado en un modelo de Lógica Difusa Compensatoria. Ingeniería industrial, vol. 36, no. 1, 2015, pp. 59.

8 HENRÍQUEZ LARRARTE, R.; BURGOS, O.; IGNACIO, R. Implicancias de una Responsabilidad Social Empresarial Sustentable. Revista Electrónica Gestión de las Personas y Tecnologías, vol. 8, no. 23, 2015, pp. 18. 
Las definiciones ofrecidas anteriormente coinciden en cuanto a los elementos a considerar en la determinación de la Responsabilidad Social Empresarial, entre estos se destacan: objetivos, intereses de los grupos sociales que define, ámbitos de actuación, dimensiones, su contribución al desarrollo de la sociedad así como a la preservación del medio ambiente y sustentabilidad de las futuras generaciones.

Otros investigadores tales como (COMISIÓN EUROPEA9; DE LA CUESTA GONZÁLEZ y MARTÍNEZ ${ }^{10}$ y SAGEBIEN Y BETANCOURT $A B I{ }^{11}$, coinciden en que la Responsabilidad Social Empresarial ha sido concebida como:

- Fuente de ganancias competitivas.

- Factor estratégico.

— Instrumento que contempla el desempeño económico, social y medioambiental.

- Elemento clave para la competitividad.

- Herramienta de gestión.

- Ventaja competitiva.

- Tema de creciente demanda social.

- Proceso que se vincula con el concepto de «sostenibilidad», que abarca a un mismo nivel aspectos económicos, sociales y medioambientales.

- Parte de la planificación estratégica.

- Aspecto que se debe incluir en la formación tanto de los directivos como de los trabajadores.

- Función clave a la hora de contribuir al desarrollo sostenible y reforzar al mismo tiempo el potencial innovador y la competitividad.

- Aspecto que alcanza efectos positivos en el incremento de los resultados económicos y en la reducción de los costos.

- Relación ética y transparente de la empresa con todos los públicos.

- Mecanismo que juega un rol en el establecimiento de metas empresariales que impulsan el desarrollo sostenible de la sociedad.

9 COMISIÓN DE LAS COMUNIDADES EUROPEAS (CCE). Libro Verde: Fomentar un marco europeo para la responsabilidad social de las empresas. Brusela: CCE, 2001.

10 DE LA CUESTA GONZÁLEZ, M.; MARTíNEZ, C.V. Responsabilidad social de la empresa. Concepto, medición y desarrollo en España. Boletín económico de ICE, no. 2755, 2003, pp. 7.

11 SAGEBIEN, J.; BETANCOURT ABIO, R. Non-state socially responsible enterprises: The key to inclusive economic growth in Cuba. En No more free lunch. Springer, Cham, 2014, pp. 193-222. 
En relación a las dimensiones de la RSE coinciden diferentes criterios entre los que se mencionan (COMISIÓN EUROPEA ${ }^{12}$; DE LA CUESTA GONZÁLEZ y MARTíNEZ ${ }^{13}$ y MONEVA ABADÍA ${ }^{14}$ ) que asumen una triple dimensión expresada a través de los aspectos económicos, sociales y medioambientales, constituyendo estas hilo conductor de los resultados de la investigación.

Los autores antes mencionados concuerdan con las siguientes definiciones:

- Responsabilidad económica. Esta dimensión implica la creación de valor para el accionista o propietario; para el cliente por medio de la atención de sus demandas; para los proveedores pagando precios justos por sus productos o servicios, y para los empleados protegiendo y generando empleo, en base a un sistema de justicia salarial, proporcionando beneficios sociales, formación, estabilidad y motivación.

- Responsabilidad social. Supone el respeto a las leyes, costumbres y cultura, así como implicarse en el ámbito político de su entorno. Los dos primeros aspectos requieren que la empresa acepte los estándares legales y socioculturales de la sociedad en la que operan, mientras que el último supone un papel más activo de la empresa, adoptando iniciativas que mejoran el bienestar general de la sociedad.

- Responsabilidad medioambiental. Implica que las empresas deben satisfacer las necesidades actuales de sus grupos de interés sin comprometer las de las generaciones futuras, contribuyendo así al desarrollo sostenible.

Los debates en torno a la Responsabilidad Social Empresarial transitan por dos variantes diferentes. Por un lado, investigaciones apuntan al papel de la empresa en la autorregulación y asimilación voluntaria y consciente de este proceso y por otra parte quienes consideran que el proceso debe ser regulado desde el Estado, quien deberá establecer las políticas que garanticen el cumplimiento de los objetivos sociales. Es válido destacar que el estudio realizado como parte de esta investi-

12 COMISIÓN DE LAS COMUNIDADES EUROPEAS (CCE). Libro Verde. Fomentar un marco europeo para la responsabilidad social de las empresas. Brusela: CCE, 2001.

13 DE LA CUESTA GONZÁLEZ, M.; MARTíNEZ, C. V. Responsabilidad social de la empresa; concepto, medición y desarrollo en España. Boletín Económico de ICE, no. 2755, 2003, pp. 7-19.

14 MONEVA ABADÍA, J. M. Información sobre responsabilidad social corporativa: situación y tendencias. RAE: Revista Asturiana de Economía, no. 34, 2005, pp. 52. 
gación, evidencia que ha primado la voluntariedad de las empresas en dicho proceso, sin dejar de mencionar que en los últimos años, los gobiernos se vienen involucrando en mayor o menor medida en este aspecto como parte de su gestión, facilitando y promoviendo el comportamiento de las empresas en tal sentido.

La existencia de disímiles criterios sobre la Responsabilidad Social Empresarial permite que esta pueda ser concebida y evaluada por las empresas en la manera que mejor se adecue el aporte que las mismas realizan a la sociedad, a partir de lo planificado en la estrategia empresarial.

Se considera que «para instituir una política de RSE y establecer el liderazgo necesario para implementarla, una organización no solo debe realizar acciones concretas o desarrollar ciertas prácticas, sino que debe llevarla más allá, e incorporar políticas de RSE como parte de la visión y misión, y de sus prácticas diarias» ${ }^{15}$.

A la par de la aplicación de las políticas de RSE, las organizaciones deberán garantizar los mecanismos para la medición de los resultados que se alcanzan a partir de su implementación.

El análisis de las definiciones estudiadas de Responsabilidad Social Empresarial, permite conocer los más variados elementos que caracterizan este proceso, sin embargo muy pocas investigaciones definen la manera de llevarla a la práctica en las organizaciones y empresas.

En general la Responsabilidad Social Empresarial debe sustentarse en los valores expresados por la empresa y debe ser plasmada en un conjunto integral de políticas, prácticas y programas a lo largo de las operaciones empresariales para institucionalizarla. De lo contrario, se caería en el riesgo de implementar prácticas que, si bien son socialmente responsables, al no responder a un mandato y cultura institucionales, están en peligro de suspenderse ante cualquier eventualidad, coyuntura, crisis presupuestal o cambio en la dirección de la empresa. Un elemento adicional fundamental es que la RSE debe ser apoyada e incentivada por los altos mandos de la organización ${ }^{16}$.

La Responsabilidad Social Empresarial lleva a la actuación consciente y comprometida de mejora continua, medida y consistente que permite a la empresa generar valor agregado para todos sus públicos, y con ello ser sustentablemente competitiva.

15 HENRÍQUEZ LARRARTE, R.; BURGOS, O.; IGNACIO, R. Implicancias de una Responsabilidad Social Empresarial Sustentable. Revista Electrónica Gestión de las Personas y Tecnologías, vol. 8, no. 23, 2015, pp. 25.

16 CAJIGA CALDERÓN, J. F. El concepto de Responsabilidad Social Empresarial RSE. Centro Mexicano para la filantropía, 2015, pp. 4-5 


\section{La Responsabilidad Social Cooperativa}

La sociedad exige cada vez más a las empresas compromisos sociales y medioambientales que superan el ámbito estrictamente económico. Por ello, a las empresas, y en particular las de la Economía Social y Solidaria, se les pide que adopten prácticas de Responsabilidad Social Empresarial dirigidas a satisfacer las expectativas de sus diferentes grupos de interés, máxime cuando las mismas pueden explicar, en buena medida, el logro de ventajas competitivas ${ }^{17}$.

Si los valores de las ESS hacen a estas organizaciones especialmente sensibles a las cuestiones relacionadas con la igualdad de género, no es menos cierto que el paralelismo existente entre estos mismos valores y los que sustentan la RSE justifican que las ESS sean especialmente proclives a adoptar comportamientos socialmente responsables ${ }^{18}$.

La Confederación Empresarial Española de la Economía Social (CEPES), sostiene que «no es ajeno a la Economía Social la RSE como estrategia empresarial, ya que entre los valores de las empresas de Economía Social y los valores que promueve la RSE existen coincidencias significativas» ${ }^{19}$.

Uno de principales problemas que a la vez ha devenido reto para el sector cooperativo, lo ha constituido la pérdida de su naturaleza y esencia. Las cooperativas cubanas no han estado exentas de esta problemática.

La definición de los objetivos sociales de las empresas cooperativas cubanas ha estado condicionado históricamente por la esencia del sistema político imperante en el país, respondiendo los mismos, más al compromiso y los aportes económicos asumidos con el Estado cubano que al proceso consciente y planificado de evaluación del impacto que su actividad económica tiene en el medio social y ambiental.

La cooperativa a su vez, debe ser capaz de asegurar y posibilitar la acción asociativa, que permita la creación de espacios y mecanis-

17 ARCAS LARIO, N.; BRIONES PEÑALVER, A.J. Responsabilidad Social Empresarial de las Organizaciones de la Economía Social. Valoración de la misma en las empresas de la Región de Murcia. CIRIEC-España, Revista de Economía Pública, Social y Cooperativa, vol. 65, 2009, pp. 143-161.

18 ARCAS LARIO, N.; BRIONES PEÑALVER, A.J. Responsabilidad Social Empresarial de las Organizaciones de la Economía Social. Valoración de la misma en las empresas de la Región de Murcia. CIRIEC-España, Revista de Economía Pública, Social y Cooperativa, vol. 65, 2009, pp. 143-161.

19 CEPES (2009): Anuario 2007-2008 de la Confederación Empresarial Española de Economía Social. En http://www.cepes.es/media/docs/Anuario_CEPES_2008_Baja_Res. pdf (11/06/2009). 
mos para poder intervenir en la planificación, organización, ejecución y evaluación de las tareas derivadas de sus objetivos y fines sociales tal y como lo realiza en su actividad económica. Por otra parte, están presentes los objetivos y tareas que podrán responder a intereses personales o de determinado grupo. Por tanto, una condición para la responsabilidad social está dada por la participación real de sus miembros en el proceso, para lo cual se requiere de un proceso educativo que además de lograr la formación económica de los asociados y demás trabajadores, profundice y logre una formación ética e ideológica basada en los valores del cooperativismo ${ }^{20}$.

La Responsabilidad Social en las empresas cooperativas deberá sustentarse tanto en sus particularidades como en un conjunto de elementos derivados de su esencia y naturaleza, tales como: 1) el reconocimiento de la conjugación de sus objetivos económicos y sociales, 2) el reconocimiento de la Responsabilidad Social como parte del proceso de gestión integral de las cooperativas, 3) la necesidad de contar con un instrumento que permita la planificación y evaluación de la Responsabilidad Social, 4) la determinación de las necesidades sociales internas y externas de la cooperativa en correspondencia con el alcance de la Responsabilidad Social, 5) la realización del diagnóstico de la situación social interna y externa de la cooperativa, 6) la evaluación de la situación social y de la Responsabilidad Social.

La Responsabilidad Social Cooperativa (RSCoop), se deriva de la propia esencia del concepto de cooperativa, así como de su doble finalidad o carácter dual, que define a este tipo de organización, como ya se ha planteado, como entidades regidas por objetivos económicos y objetivos sociales, constituyendo los primeros, una condición imprescindible y al mismo tiempo limitante para el logro de los segundos. En consecuencia, la Responsabilidad Social Cooperativa tiene como una de sus condiciones básicas, la que se ha denominado soporte material de la RSCoop ${ }^{21}$.

Esto significa, que las empresas cooperativas deberán asegurar el cumplimiento de su compromiso social, sobre la base de su desempeño

20 ALFONSO ALEMÁN, J.L. Modelo de Gestión de la Responsabilidad Social Cooperativa Directa. Estudio de caso: Cooperativa de Producción Agropecuaria «Camilo Cienfuegos». Pinar del Río. Tesis en opción al grado científico de Doctor en Ciencias Económicas. Universidad de Pinar del Río, 2008, pp. 103-104.

21 ALFONSO ALEMÁN, J.L. Modelo de Gestión de la Responsabilidad Social Cooperativa Directa. Estudio de caso: Cooperativa de Producción Agropecuaria «Camilo Cienfuegos». Pinar del Río. Tesis en opción al grado científico de Doctor en Ciencias Económicas. Universidad de Pinar del Río, 2008, pp. 40-43. 
económico y financiero principalmente, a partir de recursos propios generados como excedentes de su gestión económica (utilidades), así como de otros que se obtengan como resultado de asignaciones estatales con este fin o de gestiones específicas con otras instituciones y organismos, por ello constituye una condición básica y al mismo tiempo limitante de la Responsabilidad Social Cooperativa.

Otro importantísimo soporte de la Responsabilidad Social Cooperativa en opinión del mismo autor, está dado por la formación ética e ideológica de sus directivos, asociados y demás trabajadores sobre la base de procesos educativos que tributen a la formación de valores, desarrolladores de una conciencia solidaria, humanista; así como a la preparación técnica y profesional del recurso humano de las organizaciones como base para el desarrollo eficiente y eficaz de todos los procesos: soporte ético-ideológico.

Desde el punto de vista del soporte legal de la Responsabilidad Social Cooperativa, aun cuando los marcos legales no establecen como norma la obligatoriedad de su cumplimiento, los estatutos y demás reglamentos internos de las cooperativas deberán definir las condiciones y obligaciones al respecto 22 .

Considerando estos tres elementos condicionantes de la Responsabilidad Social Cooperativa, esta puede ser definida como el compromiso obligatorio y consciente que tiene la cooperativa de contribuir con el desarrollo y el mejoramiento de la calidad de vida de sus asociados y trabajadores, sus familias, la comunidad y la sociedad en general, sobre la base de procesos económicos eficientes y eficaces ${ }^{23}$, concepción asumida por el autor como base para el diseño del plan de acciones que se propone.

En nuestra región, muchos países han trabajado en el diseño de instrumentos de evaluación de la Responsabilidad Social Cooperativa. Entre las propuestas estudiadas se destacan:

- Ley sobre Balance Social propuesta en 1977 por parte de la Asamblea Nacional de Francia.

- La experiencia teórica desarrollada por instituciones y especialistas de España: Propuesta de contenidos de un Balance So-

22 ALFONSO ALEMÁN, J.L. Modelo de Gestión de la Responsabilidad Social Cooperativa Directa. Estudio de caso: Cooperativa de Producción Agropecuaria «Camilo Cienfuegos». Pinar del Río. Tesis en opción al grado científico de Doctor en Ciencias Económicas. Universidad de Pinar del Río, 2008, pp. 42.

23 ALFONSO ALEMÁN, J.L. Modelo de Gestión de la Responsabilidad Social Cooperativa Directa. Estudio de caso: Cooperativa de Producción Agropecuaria «Camilo Cienfuegos». Pinar del Río. Tesis en opción al grado científico de Doctor en Ciencias Económicas. Universidad de Pinar del Río, 2008, pp. 42. 
cial para aplicar a las cooperativas vascas solicitado por el Consejo Superior de Cooperativas de Euskadi al Instituto de Estudios Cooperativos de la Universidad de Deusto realizado por la Dra. Atziber Mugarra Elorriaga y la Lic. Elena Blanco Meruelo, en el mes de diciembre de 2003; Argentina: Balance Social Cooperativo Integral, un modelo argentino basado en la Identidad Cooperativa de las autoras Liliana Fernández Lorenzo, Norma Geba, Verónica Montes y Rosa Schaposnik, en el mes de agosto de 1998.

- Pacto Global. Organización de las Naciones Unidas. 8.700 entidades entre empresas y otras organizaciones, financieras y no financieras, en más de 130 países.

- 10 principios referidos a los derechos humanos, el trabajo y el medio ambiente; Cuestionario desarrollado por la Escuela de Economía Social de Andalucía en el año 2012 definidos a partir de: a) Guía G 3.1 de Global Reporting Iniciative, b) Forética Gestión Ética; SGE 21, c) Pacto Global de Naciones Unidas, d) SA8000, e) Familia de la ISO 9000 «gerencia de la calidad», f) Familia de la ISO 14000 «gerencia ambiental», g) Cuestionario de concienciación de la Comisión Europea.

- Libro Verde de la Unión Europea. Promover la responsabilidad social empresaria, tanto en Europa como a nivel internacional. Inglaterra implementó un ministerio para ello. Sus empresarios crearon «La Empresa en la Comunidad» presidida por el príncipe de Gales.

- Norma ISO 26000.

- Memorias de Sostenibilidad (documentos que engloban temas tanto económicos como sociales y medioambientales).

- Marco Conceptual de la Responsabilidad Social Corporativa elaborado por la Asociación Española de Contabilidad y Administración de Empresas (AECA).

- Proyecto Balance Social Cooperativo impulsado por la Alianza Cooperativa Internacional, sede América.

- Guía para la elaboración de Memorias de Sostenibilidad del Global Reporting Initiative (GRI).

- Informe de Balance Social Cooperativo elaborado a partir de una herramienta Cooperativas de las Américas y la DGRV como resultado del cual se certificó el Balance Social Cooperativo de 10 Cooperativas de Ahorro y Crédito peruanas.

- Modelo de Balance Social propuesto por HISPACOOP (Confederación Española de Cooperativas de Consumidores y Usuarios). 
En el año 2000, se comienzan a realizar por parte de investigadores del Centro de Estudios sobre Desarrollo Cooperativo y Comunitario (CEDECOM), de la Universidad de Pinar del Río, trabajos en función del rescate de la esencia y naturaleza cooperativa, como parte de los cuales se diseñan e implementan instrumentos para la evaluación de la Responsabilidad Social Cooperativa.

Las cooperativas agropecuarias cubanas han constituido campo de actuación para el diseño e implementación de instrumentos de RSCoop, estas garantizan la plena participación de sus integrantes en sus procesos de gestión integral, haciendo énfasis en la práctica consciente de su Responsabilidad Social, por lo que en sí significa el rescate de la esencia y naturaleza cooperativa y su implicación en el desarrollo del sector.

De igual modo, la eficiencia económica de la producción agropecuaria cooperativa, el aumento de la productividad y sus rendimientos, deberán ser los objetivos sobre el cumplimiento de los cuales se logren niveles de utilidades que sustenten financieramente los diferentes proyectos sociales que desarrolla la entidad. Esta condición para la Responsabilidad Social, está muy vinculada al proceso de distribución de las utilidades, que está regido actualmente por el Decreto-Ley 365/2018.

Por ello, se impone redefinir un marco regulatorio para la distribución de utilidades que garantice el cumplimiento de su Responsabilidad Social, y que además imponga la obligación a las entidades del sector cooperativo de aplicar instrumentos evaluativos de su desempeño social a partir de los cuales se generen las informaciones parciales y finales correspondientes a presentar a la Asamblea General de Asociados y demás órganos del Estado24.

En el sector agropecuario en particular, la práctica de la Responsabilidad Social Cooperativa deberá contribuir al logro de los objetivos sociales de la organización, así como al incremento sostenido de la calidad de vida de sus trabajadores, al mejoramiento de sus condiciones laborales, a la elevación de su sentido de pertenencia o compromiso para con la organización, lo que permite contrarrestar significativamente la desfavorable situación que presenta la fuerza de trabajo de dicho sector.

En el caso de las Cooperativas no Agropecuarias, aunque estas presentan un corto período de funcionamiento, se debe señalar, que a pesar de que se han presentado problemas en el proceso que se lleva a cabo relacionado con: la etapa de constitución, la cultura cooperativa,

24 ALFONSO ALEMÁN, J. L. Modelo de Gestión de la Responsabilidad Social Cooperativa Directa. Estudio de caso: Cooperativa de Producción Agropecuaria «Camilo Cienfuegos». Pinar del Río. Tesis en opción al grado científico de Doctor en Ciencias Económicas. Universidad de Pinar del Río, 2008, pp. 104. 
la contratación económica, entre otros; investigaciones realizadas por el Centro de Estudios sobre Desarrollo Cooperativo y Comunitario (CEDECOM) de la Universidad de Pinar del Río, en un grupo de CNA, demuestran que se inicia todo un proceso de consolidación de los indicadores económicos, productivos y sociales de estas empresas, que garantizan un incremento en los anticipos y en la distribución de las utilidades, se percibe mayor sentido de pertenencia de los socios, mejor calidad de los servicios, incorporan la proyección hacia la comunidad donde se encuentran insertadas, como resultado del cumplimiento de su responsabilidad social y de su sostenibilidad como forma empresarial.

Al igual que en el caso de las cooperativas agropecuarias, la Responsabilidad Social en las Cooperativas no Agropecuarias se sustenta desde el punto de vista económico, en el proceso de distribución de las utilidades, regido en este caso por el Decreto-Ley 366/2018.

La Responsabilidad Social Cooperativa implica un proceso integral, gradual y continuo en las cooperativas, se sustenta en los valores y principios esenciales del cooperativismo lo cual le permite enfrentar los desafíos que imponen las condiciones actuales en aras de lograr un desarrollo equitativo del sector.

Una premisa determinante para la gestión de la responsabilidad social, está dada por el reconocimiento y apoyo estatal a las empresas cooperativas, que no solo incluya el aseguramiento de los recursos materiales necesarios para este proceso, sino la adopción de instrumentos legales que despojen del carácter voluntario u opcional que presenta el mismo actualmente.

\section{Procedimiento para la realización de la Responsabilidad Social Cooperativa}

El proceso de Responsabilidad Social que acometen las cooperativas se corresponden con las necesidades sociales manifestadas por asociados, directivos, trabajadores, sus familias y miembros de la comunidad donde las mismas se encuentran insertadas, y el mismo es planificado a partir de un proceso de concertación en el que participan todos los actores implicados en el mismo.

Este proceso es sometido a valoración por parte de los asociados, directivos, trabajadores, familiares y miembros de la comunidad a través de técnicas de participación grupal lo que permite que las acciones que se determinan como parte del proceso de Responsabilidad Social Cooperativa constituyan una propuesta lo más objetiva posible y adecuada al entorno de la cooperativa. 
Este instrumento toma en consideración el plan de desarrollo de la cooperativa y contribuye en el caso cubano al desarrollo de sectores claves tales como: educación, cultura, salud, transporte, medio ambiente y capacidad institucional. Además define un conjunto de valores, principios y objetivos que sobre la base del estudio teórico realizado conducen el proceso de RSC en función de la solución de problemas socioeconómicos, medioambientales y de competitividad.

El procedimiento proyecta la práctica de la Responsabilidad Social Cooperativa en tres dimensiones fundamentales y en correspondencia con posiciones y criterios asumidos como parte del análisis realizado con anterioridad: económica, social y medioambiental.

Constituye aspecto fundamental, el cumplimiento de un conjunto de premisas que contribuyen a la práctica del proceso de RSCoop. Entre estas se definen: a) el financiamiento material, b) los procesos de formación y capacitación y c) el reconocimiento legal.

Los principios que norman el funcionamiento del procedimiento son: la mejora continua, la participación, el enfoque de proceso y la responsabilidad legal.

El procedimiento diseñado e implementado en cooperativas cubanas en función de la práctica de la RSCoop, consta de 3 etapas y 6 pasos y se detalla a continuación:

\section{Etapa I. Preparación previa}

Objetivo. Lograr la preparación requerida de los asociados y directivos para la implementación de la Responsabilidad Social Cooperativa.

\section{Paso 1. Proceso de SensibiLIZACIÓN}

Como parte de la preparación previa se realizan un conjunto de actividades (talleres, conversatorios, encuentros, charlas) para asociados y directivos, resaltando la dualidad de objetivos de la empresa cooperativa, la importancia del proceso de responsabilidad social y su aporte al desarrollo económico y social de la sociedad.

Las acciones de sensibilización se diseñan y ejecutan a partir de los roles que los asociados y directivos desempeñan dentro de la organización.

Paso 2. Evaluación de las premisas para la Responsabilidad Social CoOperativa

Para la implementación de la RSCoop se evalúa el cumplimiento de las premisas descritas con anterioridad. 
Etapa II. Capacitación para la implementación de la RSCoop

Objetivo. Capacitar a los directivos y asociados involucrados en el proceso de responsabilidad social, en aras de lograr una preparación adecuada desde el punto de vista ético-doctrinario, técnico-profesional e investigativo-innovador en los participantes.

\section{Paso 3. Diseño del programa de CAPACITACIÓN}

- Diagnóstico de necesidades de capacitación. Este diagnóstico está dirigido a la determinación de las necesidades de capacitación de los asociados y directivos, en las tres vertientes que caracterizan el proceso de educación cooperativa: ético-doctrinario, técnico-profesional e investigativo-innovador con el propósito de crear las competencias, habilidades y valores propios de los miembros de una entidad cooperativa.

- Diseño del programa de capacitación. A partir del diagnóstico de las necesidades de capacitación se procede a la elaboración de los programas y proyectos destinados a satisfacer la demanda de formación identificada, los cuales deben tener un fuerte componente didáctico y metodológico, sobre la base de la andragogía como la ciencia que estudia la formación de adultos, teniendo en cuenta que las entidades cooperativas se conforman por personas que ya tienen una formación básica, por tanto, los métodos a utilizar serán en función de esto. Otro de los aspectos a considerar como parte de este proceso es el tema de los beneficiarios, para lo cual se deben tener en cuenta asociados, directivos, sus familias, y la comunidad de manera que se garantice la efectividad del proceso a partir de la implicación de todos los actores.

Etapa III. Planificación, organización y ejecución de la Responsabilidad Social Cooperativa

\section{Paso 4. Creación del Comité para la Responsabilidad Social Cooperativa (CRSCOOP)}

Se propone la creación y constitución del CRSCoop, el cual se integra a la estructura organizativa de la cooperativa, determinándose las funciones y atribuciones de su competencia, dentro de las cuales están la coordinación de las acciones para la implementación de la Responsabilidad Social y la asesoría en el proceso de toma de decisiones. 
El mismo ha de estar conformado por asociados de la cooperativa, que, aprobados por su asamblea general, reúnen las condiciones de liderazgo y preparación técnica en este proceso.

Paso 5. Desarrollo de las acciones de la Responsabilidad Social

En este caso se identifican dos factores principales:

- Análisis del programa de desarrollo de la cooperativa de manera que se logre incorporar la Responsabilidad Social Cooperativa sustentada en la propuesta de programas y acciones lográndose un enfoque integral de este proceso.

- Definir los mecanismos y relaciones funcionales para la implementación efectiva de la RSCoop.

Etapa IV. Evaluación, control y retroalimentación

Objetivo. Evaluar el proceso, por etapas y pasos, de forma tal que permita los ajustes necesarios durante la aplicación del mismo, así como la toma de decisiones para su perfeccionamiento en futuras aplicaciones.

Paso 6. MEDición, EVALUACIÓN Y RETROALIMENTACIÓN DE LOS RESULTADOS

El proceso de medición del impacto del proceso de Responsabilidad Social se lleva a cabo a través de la valoración cualitativa, resultante de un sistema de evaluación de impactos de dicha responsabilidad, con la aplicación de instrumentos (encuestas y entrevistas) a los asociados, directivos, sus familias y la comunidad.

Además, se realizan espacios de diálogos previamente concebidos en la etapa III, tales como: reuniones, debates, charlas, talleres, encuentros, con el fin de propiciar que los actores implicados en el proceso puedan trabajar por mejorar sus propias prácticas, lo que garantiza la retroalimentación.

\section{Limitaciones y resultados de la Responsabilidad Social Cooperativa en Cuba}

El procedimiento propuesto para la realización efectiva de la RSCoop, permite ofrecer a juicio de la autora, las principales limitacio- 
nes y resultados que se presentan en la práctica de la RSCoop, en una representatividad del sector cooperativo en Cuba.

Haciendo un análisis sobre las limitaciones de la Responsabilidad Social Cooperativa en Cuba, se pudiera plantear que aún es insuficiente la materialización de la RSCoop en correspondencia con las oportunidades que este proceso ofrece en función del desarrollo económico y social.

La RSCoop (...) presenta un conjunto de reservas que pudiera ampliar los beneficios de la inversión extranjera a las comunidades y ecosistemas naturales, de la inserción en las cadenas globales de valor, así como contribuir de manera rápida a los esfuerzos por lograr seguridad alimentaria y sustitución de importaciones $(\ldots)^{25}$.

Las principales limitaciones que aun presenta la práctica de la Responsabilidad Social Cooperativa en Cuba son:

1. Insuficiencias en los procesos de formación cooperativa. Los espacios de formación y capacitación desarrollados en el sector cooperativo aún se centran en la preparación técnico-especializada, abordando en menor medida los aspectos básicos de la dimensión social y filosófico-doctrinario. Además estos espacios se reducen a aquellas cooperativas que de manera voluntaria han mostrado mayor nivel de voluntad y compromiso con este proceso.

2. Alta prioridad, en las asambleas de asociados, a la gestión económica-financiera de la entidad. Las Asambleas Generales de Asociados centran su análisis en los resultados económico-financieros reforzando el carácter economicista de las cooperativas, sin promover la práctica de la RSCoop, como parte de su proceso de gestión integral.

3. Las acciones sociales que desarrollan las cooperativas no forman parte de su proceso de gestión, sino que constituyen acciones espontáneas. Aun el sector cuenta con cooperativas que no desarrollan la RSCoop desde la instrumentación de herramientas que garanticen su adecuada planificación, ejecución y control.

4. El marco legal del sector cooperativo, no establece la obligatoriedad del cumplimiento de la responsabilidad social, aun cuando esta se contempla dentro de los principios cooperativos. El marco legal del sector cooperativo en Cuba, no esta-

25 BETANCOURT ABIO, R. La responsabilidad social empresarial en Cuba. Revista Estudios del Desarrollo Social: Cuba y América Latina, vol. 4, no. 2, 2016, pp. 3-4. 
blecen como norma la práctica de la RSCoop, por lo que los estatutos y demás reglamentos internos de las cooperativas deberán definir las condiciones y obligaciones al respecto.

Entre los principales resultados de la práctica de la Responsabilidad Social Cooperativa en Cuba resalta que el propio objeto social de estas empresas contemplan, en alguna medida, la satisfacción de necesidades existentes en las comunidades donde se encuentran enclavadas, así como de la sociedad en general.

Dentro de las principales acciones que como parte de la práctica de la Responsabilidad Social realizan las cooperativas en Cuba se encuentran:

- Contribuyen al mantenimiento de las instalaciones donde se ofrecen los servicios de salud y educación de las localidades donde estas se insertan. Además contemplan programas que contribuyen a garantizar los servicios de agua, energía, transporte y recreación.

- Suministran productos y servicios subsidiados a los asociados, trabajadores, directivos, sus familias y los miembros de la comunidad.

- Participan en el mantenimiento y construcción de obras sociales priorizadas por el país y de viviendas.

- Desarrollan actividades educativas para niños y jóvenes en función de la formación de valores y principios cooperativos y el cuidado y la protección del medio ambiente, para garantizar la sostenibilidad de las futuras generaciones.

- Realizan actividades socioculturales que contribuyen a la promoción de la cultura y al esparcimiento de los asociados, directivos, sus familias y los miembros de la comunidad.

La práctica de la RSCoop en Cuba, permite determinar los principales aportes que la implementación de este proceso ha alcanzado:

1. Planificación de la responsabilidad social como parte de la gestión integral de las cooperativas.

2. Determinación a partir de lo que establece el marco regulatorio, de los fondos destinados al proceso de responsabilidad social cooperativa.

3. Determinación de las necesidades sociales de socios, directivos, trabajadores, familias y miembros de la comunidad desde la etapa de planificación, garantizando su participación real y efectiva.

4. Establecimiento de alianzas estratégicas con otras organizaciones e instituciones en función del cumplimiento de la RSCoop. 
5. Implementación de instrumentos de evaluación de la Responsabilidad Social Cooperativa y su impacto.

6. Presentación a las Asambleas Generales de Asociados de las cooperativas de los resultados alcanzados como parte del proceso de Responsabilidad Social.

\section{Conclusiones}

1. Las diversas definiciones desde las que se enfoca la Responsabilidad Social Empresarial estudiadas, justifican la necesidad de considerar este proceso como parte de la gestión empresarial, al incorporar en su concepción las dimensiones social y medioambiental, rompiendo con esquemas anteriores que la consideraban únicamente como un factor del desarrollo económico.

2. La Responsabilidad Social Cooperativa se sustenta en los principios que rigen el cooperativismo, se basa en las particularidades del funcionamiento y administración de las cooperativas y deberá considerar la función económica de las mismas, como el soporte material que sustente su función social y a su vez incide en el logro de un mayor compromiso de su membresía con el desarrollo de la organización.

3. El procedimiento que se implementa, a través de sus etapas y pasos en las empresas cooperativas cubanas, otorga un carácter integral al proceso de gestión cooperativa y en particular a la Responsabilidad Social Cooperativa, el mismo, permite determinar las principales limitaciones y resultados que presenta dicho proceso, que se evidencia en el desarrollo socioeconómico del país.

\section{Bibliografía}

1. Alfonso Alemán, J.L. Modelo de Gestión de la Responsabilidad Social Cooperativa Directa. Estudio de caso: Cooperativa de Producción Agropecuaria «Camilo Cienfuegos». Pinar del Río. Tesis en opción al grado científico de Doctor en Ciencias Económicas. Universidad de Pinar del Río, 2008, pp. 40-43, 103-104.

2. Antelo-González, Y. y Alfonso-Robaina, D. Análisis de la Responsabilidad Social Empresarial basado en un modelo de Lógica Difusa Compensatoria. Ingeniería industrial, vol. 36, no. 1, 2015, pp. 59.

3. Arcas Lario, N.; Briones Peñalver, A.J. Responsabilidad Social Empresarial de las Organizaciones de la Economía Social. Valoración de la misma en las empresas de la Región de Murcia. CIRIEC-España, Revista de Economía Pública, Social y Cooperativa, vol. 65, 2009, pp. 143-161. 
4. Betancourt Abio, R. La responsabilidad social empresarial en Cuba. Revista Estudios del Desarrollo Social: Cuba y América Latina, vol. 4, no. 2, 2016, pp. 3-4.

5. Cajiga Calderón, J.F. El concepto de Responsabilidad Social Empresarial RSE. Centro Mexicano para la filantropía, 2015, pp. 4-5

6. Campos lópez, S.e.; Núñez Guzmán, J.A.; García Angarica, M. Responsabilidad social empresarial en una comunidad agrícola del estado de Jalisco, México. Retos de la Dirección, vol. 12, no. 2, 2018, pp. 256-273.

7. CEPES (2009): Anuario 2007-2008 de la Confederación Empresarial Española de Economía Social. En http://www.cepes.es/media/docs/Anuario_ CEPES_2008_Baja_Res.pdf (11/06/2009).

8. Comisión de las Comunidades Europeas (CCE). Comunicación de la Comisión relativa a la responsabilidad social de las empresas: una contribución empresarial al desarrollo sostenible. Bruselas: CCE, 2002, pp. 11.

9. COMISIÓN DE LAS COMUNIDADES EUROPEAS (CCE). Libro Verde. Fomentar un marco europeo para la responsabilidad social de las empresas. Brusela: CCE, 2001.

10. De la Cuesta González, M.; Martínez, C.V. Responsabilidad social de la empresa; concepto, medición y desarrollo en España. Boletín Económico de ICE, no. 2755, 2003, pp. 7-19.

11. Henríquez larrarte, R.; Burgos, O.; Ignacio, R. Implicancias de una Responsabilidad Social Empresarial Sustentable. Revista Electrónica Gestión de las Personas y Tecnologías, vol. 8, no. 23, 2015, pp. 18-25.

12. Moneva Abadía, J.M. Información sobre responsabilidad social corporativa: situación y tendencias. RAE: Revista Asturiana de Economía, no. 34, 2005, pp. 52.

13. Mozas Moral, A.; Puentes Poyatos, R. La responsabilidad social corporativa y su paralelismo con las sociedades cooperativas. REVESCO. Revista de Estudios Cooperativos, no. 103, 2010, pp. 75-100.

14. Pérez Espinoza, J.M., Espinoza Carrión, C.; Peralta Mocha, B. La responsabilidad social empresarial y su enfoque ambiental: una visión sostenible a futuro. Revista Universidad y Sociedad [seriada en línea], vol. 8, no. 3, 2016, pp. 169-178. Recuperado de http://rus.ucf.edu.cu/

15. Sagebien, J.; Betancourt ABIO, R. Non-state socially responsible enterprises: The key to inclusive economic growth in Cuba. En No more free lunch. Springer, Cham, 2014, pp. 193-222.

16. VallaeYS, F. ¿Qué es la responsabilidad social universitaria. Nuevo León, México. Consultado en: www. cedus. cl, 2008, pp 1-2.

17. Vélez Evans, A.M. Una respuesta a la acción social, de la rentabilidad a la responsabilidad social empresarial. Revista Ciencias Estratégicas, vol. 18, no. 23, 2010, pp. 12. 


\section{Derechos de autor}

http://dec.revistas.deusto.es/

La revista Deusto Estudios Cooperativos es una revista de acceso abierto lo que significa que es de libre acceso en su integridad inmediatamente después de la publicación de cada número. Se permite su lectura, la búsqueda, descarga, distribución y reutilización legal en cualquier tipo de soporte sólo para fines no comerciales y según lo previsto por la ley; sin la previa autorización de la Editorial (Universidad de Deusto) o el autor, siempre que la obra original sea debidamente citada (número, año, páginas y DOI si procede) y cualquier cambio en el original esté claramente indicado.

\section{Copyright}

The Deusto Journal of Cooperative Studies is an Open Access journal which means that it is free for full and immediate access, reading, search, download, distribution, and lawful reuse in any medium only for non-commercial purposes, without prior permission from the Publisher or the author; provided the original work is properly cited and any changes to the original are clearly indicated. 